\title{
Privacy Modelling Computation and Management for Assisted Living within Smart Homes
}

\author{
Ismini Psychoula *, Liming Chen *, Feng Chen * \\ ${ }^{*}$ School of Computer Science and Informatics, De Montfort University, Leicester, UK \\ Email: \{ismini.psychoula, liming.chen, feng.chen\}@dmu.ac.uk
}

\begin{abstract}
Ambient Assisted Living (AAL) technologies create intelligent systems to assist the aging population for a healthier and safer life in their living environment. Such systems usually offer context-aware, personalized and adaptive services. However, these kinds of systems make extensive and intensive use of personal data, which makes privacy protection a critical issue. In this paper, we propose a framework for privacy modeling computation and management for AAL within Smart Homes. We analyze the privacy features in the smart home that affect the privacy of the users. Based on these features a metric is developed to compute the sensitivity of the collected information and consequently the potential privacy risk. A simple implementation of the proposed framework is then applied to a real world smart home living environment at Great Northern Haven, in which data were collected and the framework was evaluated. This study offers an effective and practical approach to evaluate the privacy risk of users and proposes a metric that can be used for access control and recommendation of privacy settings to the users of the AAL environments.
\end{abstract}

\section{INTRODUCTION}

Ambient Assisted Living (AAL) is a multidisciplinary field that makes use of information and communication technologies in health care systems aiming at countering the effects of a growing elderly population. It proposes ICT-based solutions to allow senior citizens to continue living at home and better selfmanage their health and daily activities. According to Cook et al. [2], there is a growing need for research focusing on Ambient Intelligence (AmI) to improve the quality of life for people with disabilities and to promote aging at home. However, at the same time, the adoption of AAL technology in people's everyday lives creates new privacy challenges [1]. The threats to privacy come from personal data aggregation and the increasing power and sophistication of data mining techniques. The magnitude of the information sources and the increasing potential to combine these sources to, for example, create a person's profile, greatly threaten individual privacy [10]. In the field of AAL, this is very important partly because of the potential fragility of the users and also because they are often not aware of the possible mischievous uses their personal information could have. In order to address these threats Privacy Enhancing Technologies (PET) [22] are being developed. Such developments are also part of new approaches that arise from the concept of AmI, which focuses on modelling, processing and modifying the context of smart spaces. Furthermore, since AAL systems focus on assisting humans in their everyday life, privacy concerns have to be taken into consideration at the beginning of the design process of such systems. In order to overcome privacy concerns, the choice of data usage has to be shifted from the service providers and developers to the individual users and offer them ways to identify the sensitivity of their data and make them aware of the risks. This is why in this paper we use the AAL context to develop methods that assess the sensitivity of information collected in Ambient Assisted Living environments as well as provide ways to classify the privacy risk of the residents, as a first step towards creating a system able to provide privacy recommendations based on the context and the user's learned preferences.

The remainder of the paper is organized as follows: Section 2 describes an overview of AAL privacy and presents related works. Section 3 describes the nature and privacy characteristics of the study. Section 4 discusses the envisioned privacy framework. Section 5 introduces the proposed model to evaluate privacy risks. Section 6 describes the use case scenario for evaluation and lastly Section 7 outlines conclusions and future work.

\section{RELATED WORK}

Ambient Assisted Living services can sense and collect sensitive data such as an actual user location, personal information, vital and medical data. The absence of privacy protection methods and approaches may cause sensitive information leakage. In the following subsections, privacy-preserving methods and their use in the Internet of Things (IoT) infrastructure are presented as well as existing cryptographic techniques for anonymization.

\section{A. Encryption Techniques}

The basic level of privacy can be achieved by using the encryption of sensitive data. Encryption prevents potential adversaries who could be listening in on the communication, from getting the information. The most well known privacy protection technique to anonymize personal data is based on $k$-anonymity [21]. In a k-anonymized dataset, each record cannot be differentiated from at least $\mathrm{k}$ - 1 other records with respect to certain identifying attributes, which guarantees that each released record relates to at least $\mathrm{k}$ records [20]. However, while the $k$-anonymity method protects against identity 
disclosure, it may fail to protect against attribute disclosure [3]. Another popular technique $l$-diversity [14] showed that $k$-anonymity cannot preserve privacy against data without diversity and adversaries that use background knowledge. The 1-diversity method says that an equivalence class has 1diversity if there are at least 1 "well-represented" values for the sensitive attribute and a table is said to have 1-diversity if every equivalence class of the table has 1-diversity. A more recent study introduced $t$-closeness and showed that $l$-diversity might not be able to prevent attribute disclosure because it does not take into account the semantic closeness of the sensitive values [12]. To address these issues $t$-closeness distributes the entries of the table in lists where the distance between the distribution of the sensitive attribute in the list and the distribution of the attribute in the table are not higher than a threshold $t$. The method of $\delta$-presence [15] aims at protecting datasets against membership disclosure. The basic idea is to model the disclosed dataset as a subset of a larger dataset that represents the adversary's background knowledge. A dataset is $(\delta \min , \delta \max )$ present if the probability that an individual from the global dataset is contained in the research subset lies between $\delta$ min and $\delta$ max. Differential privacy is an additional method introduced by Dwork [6], [7] and it ensures that the probability that a statistical query in a database will produce a given result is almost the same whether it is conducted on a database with the actual individual's information in it or on one without it.

\section{B. Access Control}

Access control solutions are mainly based on logic. Users should be the ones to decide who has access to their personal data and be able to control the flow of the information that is being shared. Despite access control, being one of the most important issues in Ambient Assisted Living, additional research is needed in order to resolve this issue. Recent work focuses on rule-based techniques, using the Web Ontology Language (OWL) to build semantically rich frameworks that can represent the context-aware environment and control the flow of information [11]. Related work includes OWL and Jess combination for privacy policies specification [8], First Order Logic approach [17]. Traditionally, privacy tools were designed to hide and confuse, in order to avoid leakage of personal information. Today there is a need for privacy tools that provoke awareness of the privacy practices of contextaware systems [16]. One of the biggest challenges is addressing the privacy concerns for the capture and later access of this information. Although these issues must be addressed in the specific design of each system, general techniques for evaluating the sensitivity of the collected information are necessary. While there is a lot of previous research that deals with ways to anonymize and apply cryptography to the data, there are no solutions that evaluate which information has more privacy risks or what the users would like to hide before applying the encryption methods. The most related work is that of Liu et al. [13] where they calculated the privacy score of information shared on social networks.

\section{SMART Home Privacy Characterization}

Many different definitions have been proposed for the concept of privacy, however, until now none of them has been universally accepted. Especially in the field of Ambient Assisted Living where modelling and processing techniques are used in ways that alter the context of homes. Therefore context is of fundamental importance to AAL system privacy. Typically, context includes any available information that can be used to describe the environment of the system as well as the system itself. However, situational aspects of AAL environments may trigger different privacy concerns for different people, showing that privacy is significantly influenced by the context [2], [9].

With the performance and monitoring of daily activities, users want to protect their personal data and to be able to select the conditions for their distribution. At the same time, they want to live independently by being able to rely on services provided by the assisted living technology. In order to ensure that privacy is protected, the concept of privacy zones is used. Based on that concept, the ambient assisted living environment is divided based on the physical aspects of the space and the different activities that are being performed at different times. The sensitivity of each activity, and consequently the information that is collected about it, varies depending on several features such as physical space, which in this study is based on room location, time, and activity. For the AAL environment, we try to create privacy-preserving models by answering the following questions:

(1) Who will have access to the data?

(2) What kind of information do they contain?

(3) Where were the data collected?

(4) When were the data collected?

(5) What is the type of data and their format?

The willingness of people to share their personal data varies greatly and depends highly on the context. That is why when designing the privacy of the system these differences should be taken into account. The design methods should involve and encourage the users' participation in the design process and allow for user choices in the sharing and reuse of the data. Identifying these features is an important step of risk analysis in order to analyze and define a privacy approach. These features will assist in defining the items that need to be protected and their level of importance. This kind of information is crucial for developing effective and viable privacy frameworks.

\section{PRivacy Management Framework}

Privacy management in AAL is a complex problem. Private data are described as those that could be used to identify one person from a group. However, its not feasible to use such 
a simple privacy definition in the AAL environment because in that context is very difficult to decide which of the data could be used or which are too sensitive. In this section, an overview of the system design and its components is presented. In Ambient Assisted Living the systems generally consist of a user/resident, smart sensors, actuators, wireless networks, ubiquitous devices and end user applications. The data are initially processed and transmitted in the smart home gateway, and then, all of the sensors and devices of the system generate the collected data and sensed information. The range of data collected in AAL varies since a lot of different types of sensors and medical devices are used. These data consist of sensitive information that unauthorized third parties might be interested in. Additionally, not only the sensed data itself but also the corresponding meta information that is collected could be sensitive.

Figure 11 illustrates the main components of the proposed privacy management framework. The process in the framework begins when data are requested by the end user applications. The requested data have to go through the Privacy Risk Detector first. The Privacy Risk detector component contains functions that calculate the sensitivity score of the requested data for each private information item, based on the privacy zones and characterization features, which indicate the privacy risk of the user if this information is leaked. Then the Privacy Management component uses logic to decide if each entry that was requested, and its contents, are allowed or denied. This is achieved by comparing the sensitivity of the information to the access level of the end user. Before the data are distributed, the Data Sharing component of the framework ensures that the data are handled properly by using anonymization techniques to any sensitive information that the end user doesn't have access to. At the end of the process, the appropriate desensitized data are shared to the end user applications.

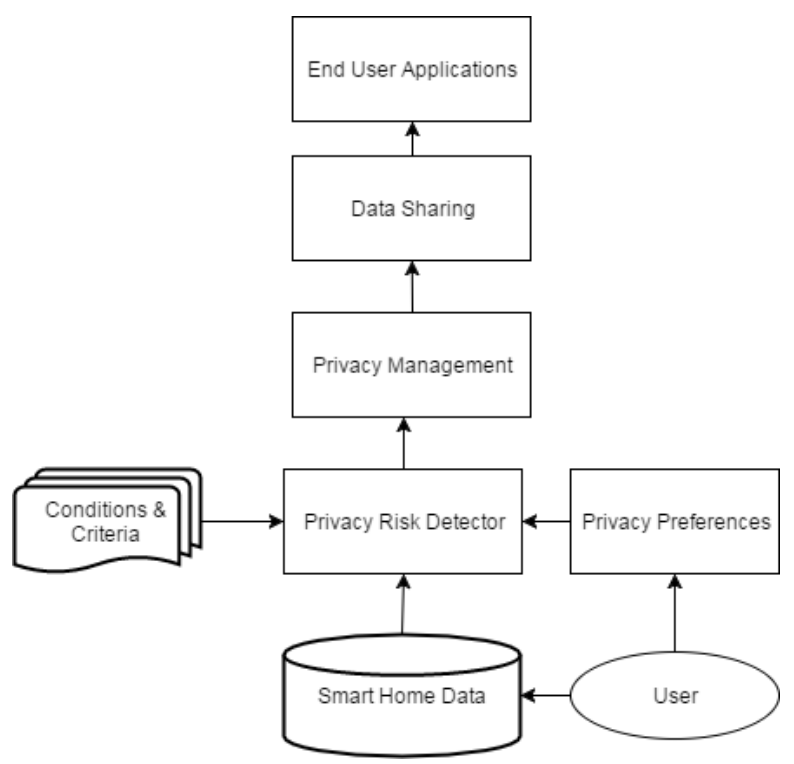

Fig. 1: Framework Architecture

\section{Smart Home Privacy Risk Detection \& SENSITIVITY METRIC}

The opinions of the users in regards to their privacy vary a lot in the field of AAL as do the devices and sensors in each smart home. For this reason, the method presented below focuses on features that are common in all AAL environments. Therefore in this method, the sensitivity of each activity, and consequently the information that is collected about it, varies depending on several features which in this study are identified as space, time, activity and type of data.

As part of the Privacy Detector component of the framework (Figure 1), the Sensitivity Metric is introduced. The Sensitivity Metric score (Equation 1) is computed as the weighted sum over a base metric which measures the sensitivity of each information item, collected and distributed from the smart home.

$$
\operatorname{SensitivityMetric}(j)=\sum_{i=1}^{n} w_{i} \times S(i, j) \times A(i)
$$

The contribution of each smart home element to the overall Sensitivity Metric score depends on the sensitivity of the information, the people that have access to it, and the user's willingness to share that piece of information. The sensitivity score of a smart home user is an indicator of their potential privacy threats. Specifically, the sensitivity score depends on:

Information item $i$ : The information item that is collected from the AAL environment.

Access $A_{i}$ : The number of end users that have access to this information increases its sensitivity.

Weight $w_{i}$ : Weight of the information item's sensitivity User $j$ : User of the smart environment

Share $S(i, j)$ : Willingness of user $j$ to share piece of information $i$.

Additive weighting in metrics is usually used for scoring and ranking alternative options based on multiple attributes. However, sometimes the step of normalizing the various attribute data in order leads to different rankings depending on which normalization method is selected. That is why a multiplication method was selected for this metric. When aggregating the criteria by using multiplication, this problem is avoided because normalization is no longer required. In addition multiplication of the Sensitivity Metric criteria allows the modelling of more realistic user preference behaviour.

\section{A. Calculating the weight of information items}

In order to calculate the sensitivity score, the sensitivity weight for all the different information items $i \in\{1, \ldots, n\}$ has to be calculated first. The weight of the sensitivity of each item is calculated using a decision matrix of criteria, where each 
criterion represents a smart home feature. A decision matrix is a special case of an information system, with a finite set of decision rules which specify what decisions should be taken when certain conditions are satisfied [19]. This provides a way to model Multiple Criteria Decision Analysis (MCDA) problems, in particular, those that have both quantitative and qualitative criteria with uncertainties. In conventional methods, an MCDA problem is modelled using a decision matrix, with each criterion assessed at each alternative decision by a single value.

The decision matrix $M(S)=\left(M_{i j}\right)$ of an information system $\mathrm{S}$ is defined as a $\gamma \times \rho$ matrix whose component at the position ( $i, j)$ is a set of attribute-value pairs. The set $M_{i j}$ contains all attribute-value pairs (attribute, value) whose values are not identical [19]. The weight refers to expressions of the relative importance of the criteria. They are generally called weights; the weight for the criterion $c \in C$ is $w_{c} \in R$. It is usually assumed that $w_{c}>0$ for all the criteria, c. Usually weights are normalized to sum to $1, \sum_{j} \in Q w_{j}=1$. Such normalization can help decision matrices to interpret the relative importance of each criterion. A weight vector is denoted $w=\left(w_{1}, w_{2}, \ldots, w_{i}\right)$, and the set of all possible weight vectors is denoted $W \subseteq R^{q}$. Most of the aggregation methods in MCDA require three steps:

1. Obtain values and weights.

2. Aggregate the values using weights.

3. Apply the aggregated values to carry out the specified task (such as choice, ranking, categorization etc.).

Let $I=\left\{i_{1}, i_{2} \cdots i_{n}\right\}$ be a set of information items collected in smart homes, which will have to be assigned weights based on various relevant criteria $C=\left\{c_{1}, c_{2} \cdots c_{m}\right\}$ and let $w_{i}$ be a weight calculated from the decision matrix having $v_{1}, v_{2} \cdots v_{m}$ as the outputs such that $w_{i}$ is the sum value of $v_{1}, v_{2} \cdots v_{m}$. The algorithm calculates the overall sensitivity weight from all the criteria with value of $w_{i}=$ $\operatorname{sum}\left(v 1, v 2 \cdots v_{m}\right)$ and assigns this weight to that information item.

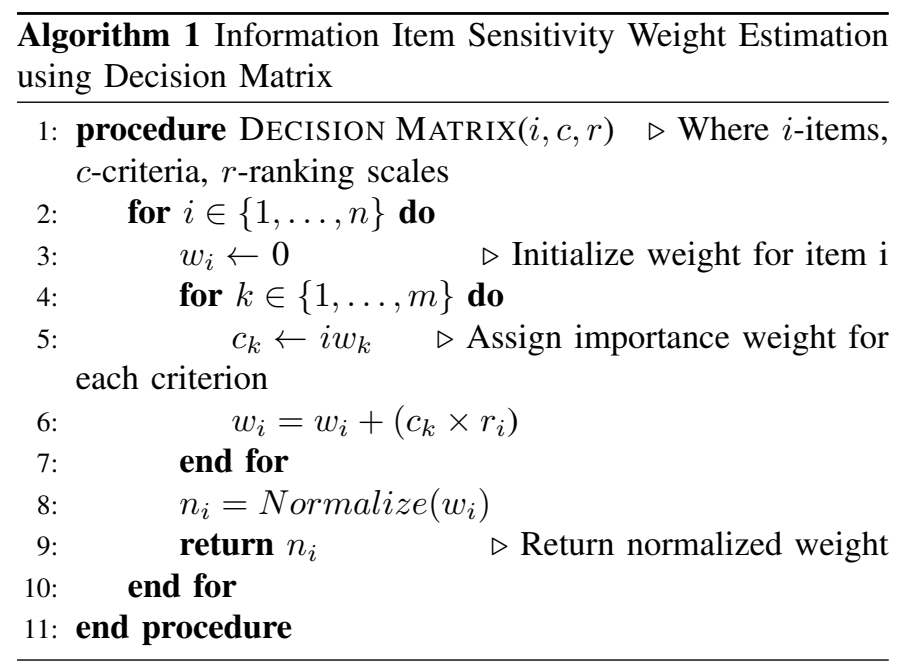

\section{CASE STUdY}

This section illustrates how the sensitivity score can be applied to a real world smart home environment. To evaluate the framework a case study was performed at Great Northern Haven (GNH) where data were collected and managed. GNH is a building complex consisting of 16 smart homes which are equipped with ambient sensors and actuators. Older adults have permanently occupied fifteen of the apartments since 2010 while the remaining apartment is used for research and demonstration purposes [5]. The existing smart home at GNH consists of environmental wired sensors including window, door, consumption usage, passive infrared (PIR) motion detectors, light and temperature sensors, as seen in Figure 2

For the evaluation of this framework and classification of the information additional sensors were deployed in the environment. The added sensors include contact sensors, sleeping mats, cameras, and flood sensor, as well as multi-sensors (as shown in Figure 2). Furthermore, wearable sensors such as smartwatches, heart rate monitors and smart cups for hydration monitoring were used to create a complete dataset. Data collection was performed for a continuous two weeks trial from two researchers.

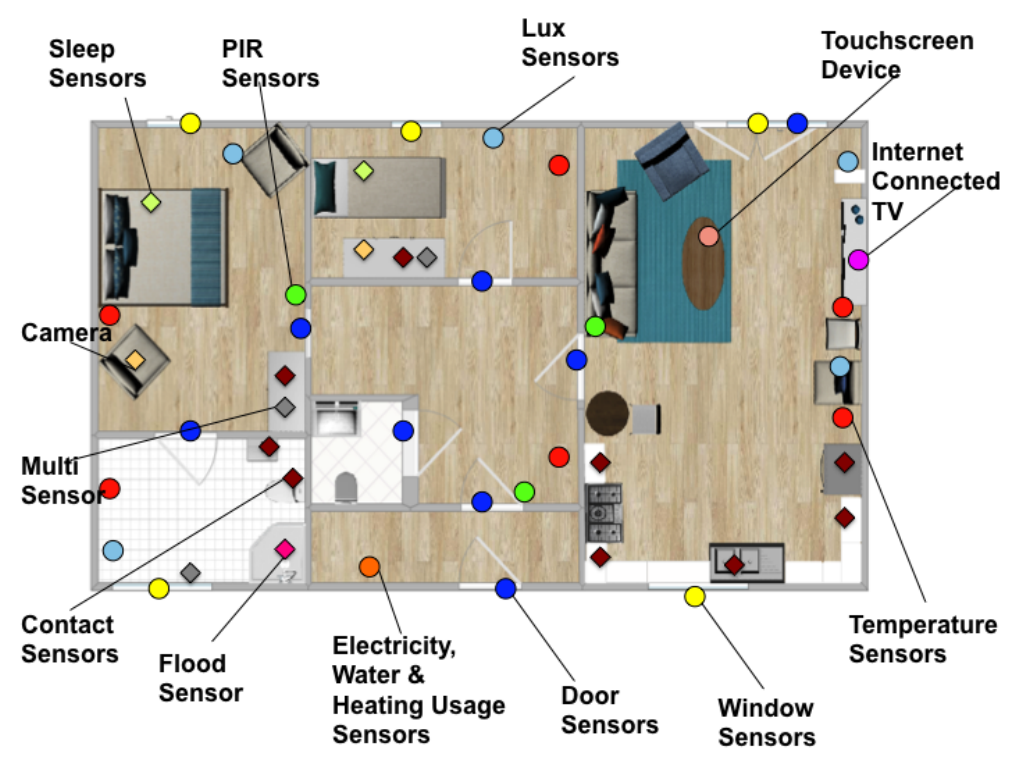

Fig. 2: Great Northern Heaven Smart Home Layout and Sensors (॰: Represents Existing Sensors and $\diamond$ : Added Sensors)

\section{A. Great Northern Haven Scenario}

In this scenario, our user Mary is one of the residents at GNH. She is 70 years old and likes to stay in close contact with her family and friends. The ambient assisted living environment she lives in allows her to live independently and gives her control over her home system through automation. For example, she can control the windows/doors, lighting, heating, and security systems along with monitoring her vital signs by a number of sensors in the apartment. These sensors 


\begin{tabular}{|c|c|c|c|c|c|c|}
\hline & Data Type $(\mathrm{EI} i w=0.4)$ & Location (VI $i w=0.3$ ) & Activity (MI $i w=0.2)$ & Time $(\mathrm{LI} i w=0.1)$ & Estimated Weight & Normalized Weight \\
\hline Blood Pressure & Moderate $(0.4 \times 2)$ & Low $(0.3 \times 1)$ & Moderate $(0.2 \times 2)$ & Low $(0.1 \times 1)$ & 1.9 & 0.63 \\
\hline Address & High $(0.4 \times 3)$ & High $(0.3 \times 3)$ & High $(0.2 \times 3)$ & Low $(0.1 \times 1)$ & 2.8 & 0.93 \\
\hline
\end{tabular}

TABLE I: Example Decision Matrix

deliver information to the cloud offering access to, family members, caregivers, doctors as well as medical health care providers, technical staff, emergency response staff and researchers.

When realizing and implementing a scenario as described above, in which potentially sensitive data collected by smart home devices are distributed, different privacy aspects have to be considered. Additionally, not only the sensed data itself but also the corresponding meta-information might be considered sensitive, especially when thinking of location privacy, where location information and timestamps are being collected from most of the sensors.

\section{B. Practical Example}

In the following, we present an example of these aspects, for the scenario of our user Mary, and a practical application of the method to calculate the sensitivity weights that will later be used to compute the Sensitivity Metric. As described previously the following features affect privacy greatly, that is why they are taken into account as criteria when calculating the sensitivity weight of information items:

1) Type of data (personal, medical, sensor based)

2) Room Location

3) Time

4) Activity

The data type describes what the types of the private data are. Room location indicates the space the sensor is placed in or the activity is taking place. Time refers to the time the information was recorded from the sensors and activity describes if any activity is being automatically detected from the sensors and stored. The procedure to collect and decide on the criteria and their weights included focus groups with residents of smart homes and discussions with experts in the domain.

Figure 3 provides an overview of the Decision Model. Each information item is described by a set of condition criteria, where $C=\{$ Location, Activity, DataType,Time $\}$, an importance level for each criterion and ranking scale for each attribute. Each of the criteria has attribute values for example for our user Mary those are:

$V$ ALlocation $=\{$ kitchen, lounge, bedroom, bathroom $\}$

$V$ ALactivity $=\{$ cooking, eating, sleeping, showering $\}$

$V A L d a t a=\{$ personal, medical, sensor $\}$,

$V$ ALtime $=\{$ morning, noon, afternoon, evening $\}$

Importance Levels $=\{$ ExtremelyImportant $(E I)$,

VeryImportant $(V I)$, ModeratelyImportant $(M I)$,

LeastImportant $(L I)\}$

and Ranking Scales $=\{$ High, Moderate, Low $\}$

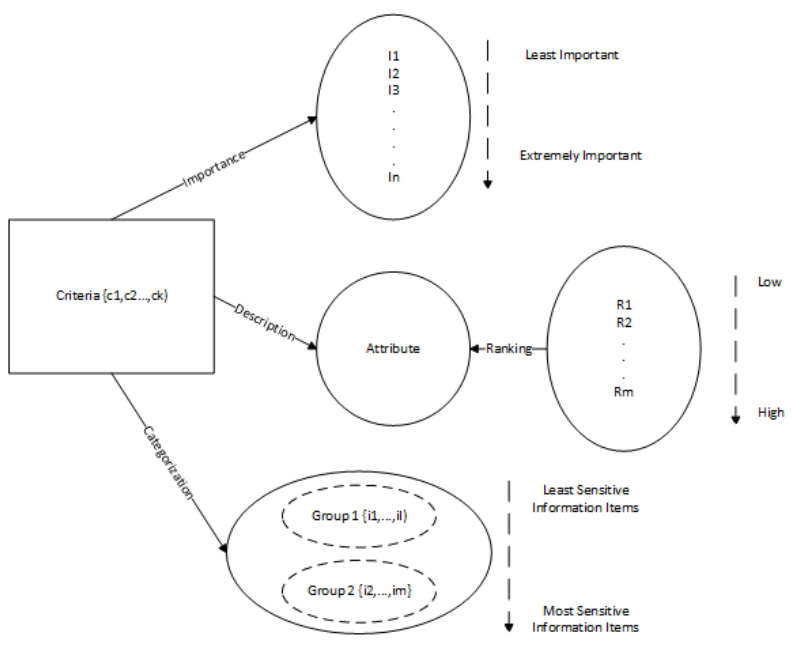

Fig. 3: Decision Model (adapted from [4])

Based on that information a weight for each information item is calculated and then they are categorized into groups based on their sensitivity.

In the example on Table I we assign initial weights to each of the criteria and we combine them with the information item's ranking scale $($ High $=3$, Moderate $=2, \mathrm{Low}=1)$ based on Mary's preferences. Naturally, information about the Address is more risky for the user than Blood Pressure and after normalization that is reflected in the overall estimated weight which is 0.63 for Blood Pressure and 0.93 for Address. The total calculated weight gives an indication of the sensitivity of the information and is then used in the computation of the Sensitivity Metric.

To compute the Sensitivity Metric the previously calculated weights are used in an example along with some additional information. In this use case scenario Mary has shared information about her blood pressure with her caregiver and her doctor, she has also shared information about her address with her daughter, caregiver and emergency response staff. Her willingness to share her blood pressure is 0.7 and her address 0.4. As shown in the simplified example below (Equation 2) the Sensitivity Metric for Mary is 1.998 .

$$
\begin{aligned}
\operatorname{SensitivityMetric}(j)= & (0.63 \times 0.7 \times 2)+(0.93 \times 0.4 \times 3) \\
& =1.998
\end{aligned}
$$

Afterwards, the metric can be utilized in the Privacy Management component of the framework as a decision making element for the sharing of the information. It will be used to give an indication of the risk that sharing this information 
could have and to recommend privacy settings depending on the risk the information poses to the user.

\section{CONCLUSION \& FutuRE WORK}

In this paper, a privacy management framework and a metric model were presented for computing the sensitivity score of information collected in smart home environments. The main focus was on sensitivity detection model and framework that will facilitate it and help to increase user acceptance and privacy awareness in the area of AAL. The proposed metric can assist in the recommendation of privacy settings by evaluating the privacy risk the information poses to the users. In the future, the feasibility of the proposed model will be validated further. In order to achieve this, prototypes are currently being developed and a participatory design approach [18] is employed.

Further work also includes a set of experiments on synthetic and real world data, as well as the expansion of the model into one where the system will be able to calculate the sensitivity score by learning the user's preferences on its own and applying them in the computation of the score. Moreover, we plan to improve the framework by implementing all the components in a more complicated use case and try to improve the efficiency for larger datasets. This work addresses the issue of privacy in smart home environments by taking into account a user-centered perspective that can help raise awareness and alert users about the sensitivity of the information that is collected and shared in their smart home environments, as well as help recommend privacy settings for them.

\section{ACKNOWLEDGMENT}

This work has been funded by the European Union Horizon2020 MSCA ITN ACROSSING project (GA no. 616757). The authors would like to thank the members of the project consortium for their valuable inputs.

\section{REFERENCES}

[1] Patrice Caire, Assaad Moawad, Vasilis Efthymiou, Antonis Bikakis, and Yves Le Traon. Privacy challenges in ambient intelligence systems. Journal of Ambient Intelligence and Smart Environments, 8(6):619-644, 2016.

[2] Diane J Cook, Juan C Augusto, and Vikramaditya R Jakkula. Ambient intelligence: Technologies, applications, and opportunities. Pervasive and Mobile Computing, 5(4):277-298, 2009.

[3] Josep Domingo-Ferrer and Vicenç Torra. A critique of k-anonymity and some of its enhancements. In Availability, Reliability and Security, 2008. ARES 08. Third International Conference on, pages 990-993. IEEE, 2008 .

[4] Michael Doumpos and Constantin Zopounidis. Multicriteria decision aid classification methods, volume 73. Springer Science \& Business Media, 2002.
[5] Julie Doyle, Andrea Kealy, John Loane, Lorcan Walsh, Brian O'Mullane, Carl Flynn, Andrew Macfarlane, Brennon Bortz, R Benjamin Knapp, and Rodd Bond. An integrated home-based self-management system to support the wellbeing of older adults. Journal of ambient intelligence and smart environments, 6(4):359-383, 2014.

[6] Cynthia Dwork. Differential privacy: A survey of results. In International Conference on Theory and Applications of Models of Computation, pages 1-19. Springer, 2008.

[7] Cynthia Dwork. Differential privacy. In Encyclopedia of Cryptography and Security, pages 338-340. Springer, 2011.

[8] Fabien L Gandon and Norman M Sadeh. Semantic web technologies to reconcile privacy and context awareness. Web Semantics: Science, Services and Agents on the World Wide Web, 1(3):241-260, 2004.

[9] Irini Genitsaridi, Antonis Bikakis, and Grigoris Antoniou. Deal: A distributed authorization language. Pervasive and Ubiquitous Technology Innovations for Ambient Intelligence Environments, page 188, 2012.

[10] Jong Hyuk Park, Stefanos Gritzalis, Ching-Hsien Hsu, Maria Karyda, Stefanos Gritzalis, Jong Hyuk Park, and Spyros Kokolakis. Privacy and fair information practices in ubiquitous environments: Research challenges and future directions. Internet Research, 19(2):194-208, 2009.

[11] Pramod Jagtap, Anupam Joshi, Tim Finin, and Laura Zavala. Preserving privacy in context-aware systems. In Semantic computing (ICSC), 2011 fifth IEEE international conference on, pages 149-153. IEEE, 2011.

[12] Ninghui Li, Tiancheng Li, and Suresh Venkatasubramanian. t-closeness: Privacy beyond k-anonymity and 1-diversity. In Data Engineering, 2007. ICDE 2007. IEEE 23rd International Conference on, pages 106-115. IEEE, 2007.

[13] Kun Liu and Evimaria Terzi. A framework for computing the privacy scores of users in online social networks. ACM Transactions on Knowledge Discovery from Data (TKDD), 5(1):6, 2010.

[14] Ashwin Machanavajjhala, Daniel Kifer, Johannes Gehrke, and Muthuramakrishnan Venkitasubramaniam. 1-diversity: Privacy beyond kanonymity. ACM Transactions on Knowledge Discovery from Data (TKDD), 1(1):3, 2007.

[15] Mehmet Ercan Nergiz, Maurizio Atzori, and Chris Clifton. Hiding the presence of individuals from shared databases. In Proceedings of the 2007 ACM SIGMOD international conference on Management of data, pages 665-676. ACM, 2007.

[16] Gautham Pallapa, Mohan Kumar, and Sajal K Das. Privacy infusion in ubiquitous computing. In Mobile and Ubiquitous Systems: Networking \& Services, 2007. MobiQuitous 2007. Fourth Annual International Conference on, pages 1-8. IEEE, 2007.

[17] Anand Ranganathan and Roy H Campbell. An infrastructure for context-awareness based on first order logic. Personal and Ubiquitous Computing, 7(6):353-364, 2003.

[18] Douglas Schuler and Aki Namioka. Participatory design: Principles and practices. CRC Press, 1993.

[19] Ning Shan and Wojciech Ziarko. Data-based acquisition and incremental modification of classification rules. Computational Intelligence, 11(2):357-370, 1995

[20] Latanya Sweeney. Achieving k-anonymity privacy protection using generalization and suppression. International Journal of Uncertainty Fuzziness and Knowledge-Based Systems, 10(05):571-588, 2002.

[21] Latanya Sweeney. k-anonymity: A model for protecting privacy. International Journal of Uncertainty, Fuzziness and Knowledge-Based Systems, 10(05):557-570, 2002.

[22] Yang Wang. Privacy-enhancing technologies. In Handbook of research on social and organizational liabilities in information security, pages 203-227. IGI Global, 2009 